\title{
Tetraphenylethene Derivatives Modulate the RNA Hairpin-G- quadruplex Conformational Equilibria in Proto-Oncogenes
}

\author{
Payal Gupta, [a] Divya Ojha, [a] Dinesh N. Nadimetla, [b] Sheshanath V. \\ Bhosale[b] and Ambadas B. Rode*[a]
}

[a] Regional Centre for Biotechnology, NCR Biotech Science Cluster, 3rd Milestone, Faridabad - Gurugram Expressway, Faridabad, Haryana 121001, India

E-mail: ab.rode@rcb.res.in

[b] Department of Chemistry, Goa University, Taleigao Plateau, Goa, India

\section{Graphical Abstract}

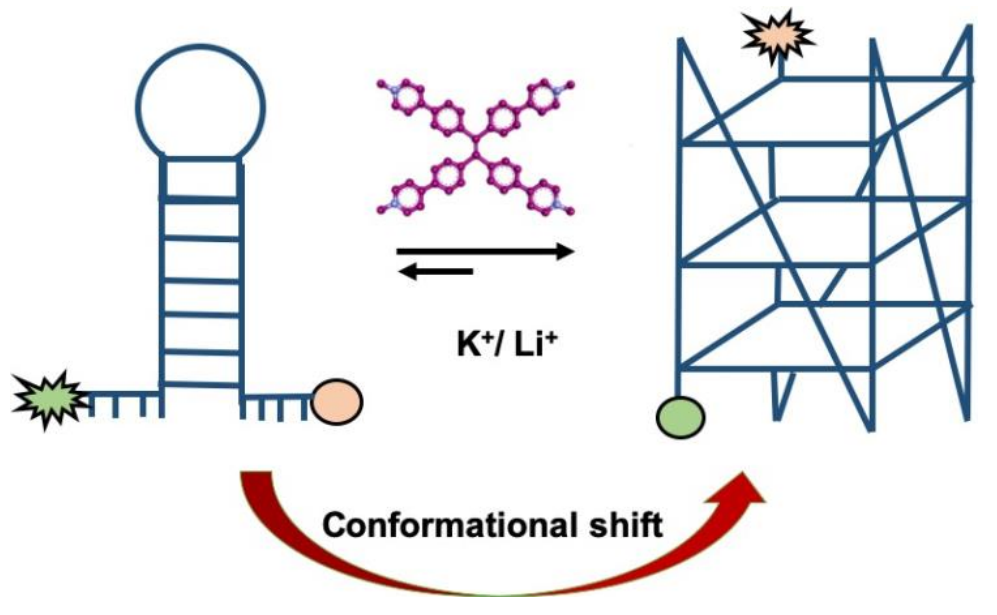

\begin{abstract}
RNA G-quadruplexes (GQs) sequence in 5' UTRs of certain proto-oncogenes colocalize with hairpin (Hp) forming sequence resulting in intramolecular Hp-GQ conformational equilibria which is suggested to regulate cancer development and progression. Thus, regulation of Hp-GQ equilibria with small molecules is an attractive but less explored therapeutic approach. Herein, two tetraphenylethenes (TPE) derivatives TPE-Py and TPEMePy were synthesized and their effect on Hp-GQ equilibrium was explored. The FRET, CD and molecular docking experiments suggested that cationic TPE-MePy shifts the Hp-GQ equilibrium significantly towards the GQ conformer mainly through $\pi-\pi$ stacking and van der waals interaction. In presence of TPE-MePy the observed rate constant values for first and second folding step was increased up to 14.6 and 2.6-fold respectively. The FRET melting assay showed a strong stabilizing ability of TPE-MePy $\left(\Delta \mathrm{Tm}=4.36{ }^{\circ} \mathrm{C}\right)$. Notably, the unmethylated derivative TPE-Py did not alter the Hp-GQ equilibrium. Subsequently, the luciferase assay demonstrated that the TPE-MePy derivatives suppressed the translation efficiency by 5.7-fold by shifting the Hp-GQ equilibrium toward GQ conformers in 5' UTR of TRF2. Our data suggest that HpGQ equilibria could be selectively targeted with small molecules to modulate translation for therapy.
\end{abstract}




\section{Introduction}

Human genome exhibits more than 3,00,000 guanine-rich regions[1] capable of forming Gquadruplex (GQ) conformations via Hoogsteen hydrogen bonds.[2] The formation of GQ in deoxyribo and ribonucleic acids regulates gene expression at transcriptional[3] and translational level[4] respectively and thus act as a potential therapeutic target.[5] It has been suggested that the RNA GQ formation is more facile owing to the absence of a competing complementary strand, thermodynamically more stable and less polymorphic than their DNA counterparts.[6] However, RNA GQ folding has been found to be regulated by certain inherent and environmental factors. The environmental factors involve cations,[7] molecular crowding agents[8] and other abundant biomolecules like tRNAs.[9] While, the inherent factors consist of flanking sequences of the $\mathrm{G}$ rich regions,[10] number of G-quartets, loop base-pair identities and loop length.[11]

Among the various inherent factors, the flanking sequences of the $\mathrm{G}$ rich region are very crucial since they can modulate the GQ folding and their regulatory functions.[9-10, 12] GQs have been observed to colocalize with hairpin (Hp) forming sequence[9-10] and in certain cases, the intramolecular conformational transitions between the two structures regulate biological functions such as protooncogene translation[9, 12b] and miRNA maturation.[12a, 13] Together, these observations suggest that the Hp-GQ conformational equilibria are promising therapeutic targets. In spite of the increasing understanding on the role of Hp-GQ conformational equilibria in certain diseases; reports on its use as therapeutic target for small molecules are scarce[14] and subsequently highlight the need to develop ligands to target specific RNA GQ functions.[15] Herein we have demonstrated for the first time that the HpGQ equilibria could be selectively targeted with small molecules to modulate translation for therapy.

We[16] and others[17] have synthesized diverse tetraphenylethenes (TPE) derivatives as Aggregation Induced Emission (AIE) probes for biosensing applications including interactions with amino acids,[18] nucleotides,[19] nucleic acids,[20] and DNA G-quadruplexes.[21] The TPE core is believed to be an attractive starting point for developing GQ binding ligand therapeutics given its strong binding due to its appropriate size and selectivity for GQ over other DNA conformations.[21b] In light of the above facts, we envisioned that TPE derivatives might modulate the RNA conformational dynamics between hairpin and GQ structures in the 
5' untranslated regions (UTRs) of certain oncogenes, ultimately impacting the cellular proliferation.

We have synthesized a tetra(4-pyridyl) tetraphenylethene (TPE-Py) as shown in Figure 1a by fusing the TPE with the pyridine rings (Figure S1a). The pyridine rings were fused to extend the $\pi$-conjugated plane of TPE that could improve the stacking interactions. Furthermore, at physiological $\mathrm{pH}$ the nitrogen atom in the pyridine can be protonated, thus it can facilitate the electrostatic interaction between the neutral TPE scaffold and the negatively charged core of the GQ. In addition, we have also synthesized a cationic tetra (N-methyl-4-pyridyl) tetraphenylethene (TPE-MePy) by methylation of TPE-Py as shown in Figure $1 \mathrm{~b}$ and S1b. The $\mathrm{N}$-methylated pyridines cationic groups in TPE can promote electrostatic interactions as well as can increase the water solubility as has been observed in case of porphyrin derivatives.[22]

a)

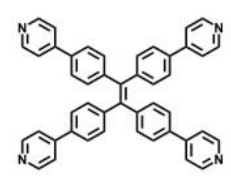

b)

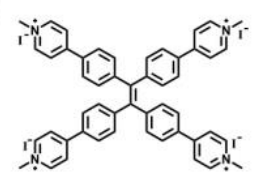

c)

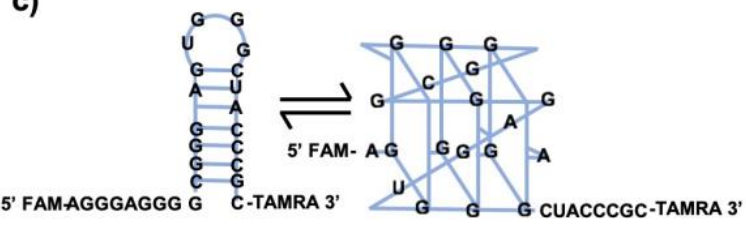

Figure 1. Chemical structure of a) TPE-Py; b) TPE-MePy; and c) Graphical representation of predicted Hp-GQ conformational equilibria adopted by RNA HpGQ-1 sequence (FAM $=6$-carboxyfluorescein; TAMRA = tetramethylrhodamine).

To evaluate the in vitro effect of TPE derivatives on the rate of hairpin-GQ conformational transitions, we have used a Fluorescence resonance energy transfer (FRET) technique. FRET has been used to detect the different conformations of nucleic acids including the GQs given its dependence on the distance and orientation of attached fluorophores.[9, 23] For this, we have chosen a RNA sequence HpGQ-1, (Figure 1c) which can adopt mutually exclusive hairpin and GQ structures and was covalently attached at 5' end with 6-carboxyfluorescein (FAM) as a donor and at 3' end tetramethylrhodamine (TAMRA) as an acceptor.[9] In the hairpin form the average distance between the two chromophores is relatively larger as compared to the GQ folded structure in which the folding brings two chromophores in close proximity to observe the energy transfer (Figure 1c). We envisioned that the TPE derivatives interaction with HpGQ 
RNA would result in change of the FRET signals and that can be monitored to evaluate its effect on the kinetics of hairpin-GQ conformational dynamics.

First, to study the conformational transition of HpGQ-1 by FRET method, we pre-formed HpGQ-1 into hairpin conformation in presence of $1 \mathrm{mM} \mathrm{MgCl} 2$. The conformational shift from hairpin to G-quadruplex was induced by addition of either $100 \mathrm{mM} \mathrm{LiCl}$ or $\mathrm{KCl}$ (Figures 2a, $2 \mathrm{~b}, \mathrm{~S} 4 \mathrm{a})$ and FRET signals were monitored as a function of time at $30^{\circ} \mathrm{C}$. We observed relatively significant increase in FRET signals in presence of $\mathrm{K}+$ as compared to $\mathrm{Li}+$ (Figure $\mathrm{S} 4 \mathrm{a})$, indicating the G-quadruplex formation in presence of $\mathrm{K}+$. The G-quadruplex formation was also confirmed by CD using non-labeled HpGQ-1 RNA oligo (Figure S4b). Next, to evaluate the effect of TPE derivatives on the conformational transition we performed the same experiments in the presence of $100 \mathrm{mM} \mathrm{KCl}$ with TPE-Py (Figure 2a) and TPE-MePy (Figure $2 \mathrm{~b}$ ) in concentration range of $60 \mathrm{nM}$ to $1 \mu \mathrm{M}$. As a result, the FRET signals were increased in the presence of both the ligand derivatives with increase in incubation time. However, in presence of TPE-Py there was no change observed in FRET signal intensities at used concentration range i.e. $60 \mathrm{nM}$ to $1 \mu \mathrm{M}$ relative to $0 \mu \mathrm{M}$ concentrations. For the clarity we have shown the FRET signals data only for the highest concentration i.e. $1 \mu \mathrm{M}$ (Figure $2 \mathrm{a}$ ). Notably, we also observed increase in FRET signal intensity in presence of LiCl and TPEMePy (Figure S5). This suggest that TPE-MePy can induce Hp-GQ conformational transition in the absence $\mathrm{KCl}$.
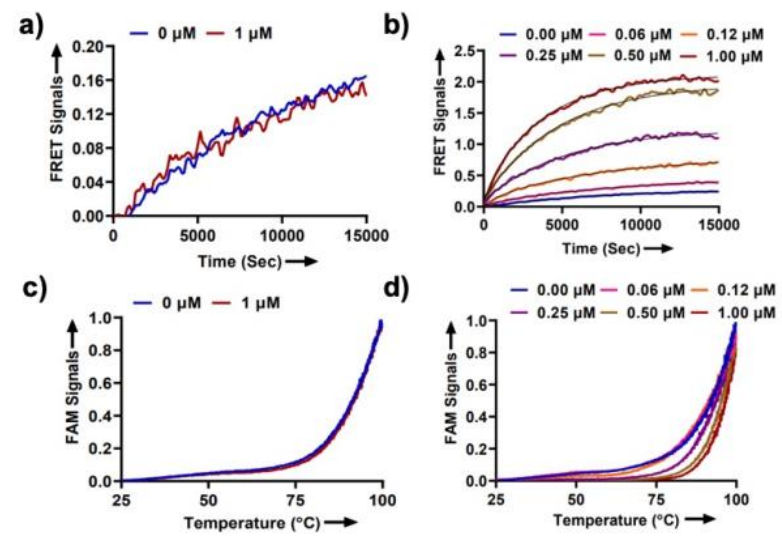

Figure 2. FRET kinetic and FRET melting profile of HpGQ-1 oligonucleotide in presence of TPE derivatives. FRET signals as a function of time after adding $\mathrm{KCl}$ and a) TPE-Py; or b) TPE-MePy as indicated. Time course of FRET signals changes after mixing $100 \mathrm{mM}$ of $\mathrm{KCl}$ and a) TPE-Py; or b) TPE-MePy as indicated in a buffer containing $30 \mathrm{mM}$ MOPS (pH 7.5), $1 \mathrm{mM} \mathrm{MgCl}_{2}$, and $0.08 \%$ Tween 20 at $30^{\circ} \mathrm{C}$. The black lines through the FRET data are the fits to double exponentials. The FAM fluorescence emission has been monitored as a function of temperature in presence of c) TPE-Py; and d) TPE-MePy in a buffer containing $10 \mathrm{mM}$ lithium cacodylate (pH $=7.2), 1 \mathrm{mM} \mathrm{KCl}, 1 \mathrm{mM} \mathrm{MgCl} 2$, and $0.08 \%$ Tween 20 at $30^{\circ} \mathrm{C}$. The plotted $\mathrm{FAM}$ emission intensity corresponds to normalized fluorescence. The FRET and melting curves are averages of triplicate experiments. 
In order to evaluate the effect of TPE-MePy on rate constants of the Hp-GQ conformational transition, we fitted the obtained FRET data (Figure 2b) to a double-exponential rate equation (Supporting Information Equation 1). The better fit of the double-exponential rate equation suggests formation of triplex-like intermediate in the GQ folding, which is consistent with the previous observations.[9] The observed rate constants for the first kinetic step (kobs1) and the second kinetic step (kobs2) are shown in Table 1. In the absence of TPE-MePy, both kobs1 and kobs 2 values obtained for HpGQ-1 are $1.33 \times 10-4 \mathrm{~s}^{-1}$ and $0.93 \times 10-4 \mathrm{~s}^{-1}$ respectively and are consistent with the previously observed values.[9] The kobs 1 and kobs2 values were found to be increased with the increase in the TPE-MePy concentration. In presence of $1 \mu \mathrm{M}$ TPEMePy we observed 14.6-fold (19.4×10-4 s-1) and 2.6-fold (2.45×10-4 s-1) increase in kobs1 and kobs2 values, respectively relative to that in absence of ligand (Table 1). The rate of GQ folding is a crucial parameter to determine its capacity to regulate function in biologically relevant timescale.[24] Our data suggest that the TPE-MePy can facilitate GQ mediated regulatory function by faster folding. The TPE-MePy might have lowered the activation energies of GQ folding by its binding with HpGQ oligo resulting in faster Hp-GQ conformational transition. The faster GQ folding rates in presence of TPE-MePy also suggest the displacement of Hp-GQ equilibrium to GQ conformer which is also evident from the significant increase in FRET signal intensities at the saturation points. The increase in FRET signal intensity as a function of TPE-MePy concentration suggests that the TPE-MePy shifts the hairpin-GQ equilibrium toward the GQ conformer. We further used FRET signals to determine the binding stoichiometry of TPE-MePy for HpGQ-1 RNA by conducting continuous variation i.e., Job's plot analysis. FRET signals of a series of solution containing different molar fraction of TPE-MePy and HpGQ-1 were measured at $25^{\circ} \mathrm{C}$ (Figure S6). The result shows 2:1 binding mode i.e. two molecules of TPE-MePy binds to one molecule of HpGQ-1 RNA.

Next, in order to assess the effect of TPE derivatives on the stability of GQ, FRET-melting assays were performed. The melting assay has been extensively used to evaluate the stabilization induced by GQ ligands based on a difference in melting temperature (Tm) between the GQ in the absence and presence of the ligands.[25] We have performed the melting assays at $1 \mathrm{mM}$ monovalent cation concentration due to high stability of HpGQ-1. After folding and incubation of HpGQ-1 RNA with TPE derivatives the FAM signal was monitored as a function of temperature (Figures 2c, d). No differences in Tm were observed in presence of TPE-Py derivative (Figure 2c), however we observed increase in Tm with increase in 
concentration of TPE-MePy (Figure 2d, Table 1). The observed stabilization by TPE-MePy derivative is shown in Table 1, in terms of the difference in the mid-transition temperature with and without the TPE-MePy ( $\square \mathrm{Tm}$ ). The TPE-MePy strongly stabilized the GQ with $\square \mathrm{Tm}$ values of $4.36 \square \mathrm{C}$ at $1 \mu \mathrm{M}$ TPE-MePy (Table 1). Notably, TPE-MePy also stabilized the GQ structures in presence of $\mathrm{LiCl}$ (Figure S7 ). This data suggest that the TPE-MePy can induce GQ even in absence of $\mathrm{KCl}$ and presence of $\mathrm{LiCl}$.

To further analyse the effect of the TPE derivatives on the Hp-GQ conformational equilibrium we have used Circular dichroism (CD) spectroscopy. CD is a very promising technique not only for the determination of the GQ structures, stability, and topology, but also for studying the interaction of ligands with GQ structures.[26] We have performed CD experiment using non-labelled HpGQ-1 RNA in the presence of $1 \mathrm{mM} \mathrm{KCl}$ or $\mathrm{LiCl}$ and with or without the TPE derivatives at $25{ }^{\circ} \mathrm{C}$. In the absence of TPE derivatives and presence of $\mathrm{KCl}, \mathrm{CD}$ spectra showed negative peaks at $210 \mathrm{~nm}$ which is a characteristic of the formation of an A-form RNA helix.[9] In addition, a positive peak at $265 \mathrm{~nm}$ and a negative peak at $240 \mathrm{~nm}$, indicates formation of parallel G-quadruplex (Figures 3a, 3b). In presence of $20 \square \mathrm{M}$ TPE-Py no differences in positions of peak or peak intensities were observed relative to $0 \square \mathrm{M}$ TPE-Py (Figure 3a), suggesting no interaction with the RNA. On the other hand in the presence of TPE$\mathrm{MePy}$, the intensities of the positive peak at $265 \mathrm{~nm}$ were increased and intensities of negative peak at $210 \mathrm{~nm}$ were decreased with increasing TPE-MePy concentration (Figure $3 \mathrm{~b}$ ). No significant differences were observed at $240 \mathrm{~nm}$ peaks intensities with or without the TPE derivatives. Notably, HpGQ-1 showed characteristic peaks of parallel G-quadruplex in presence of TPE-MePy and $1 \mathrm{mM} \mathrm{LiCl}$ (Figure S8). All these data suggest that the TPE-MePy was able to shifts the Hp-GQ equilibrium towards the GQ conformer in presence of both $\mathrm{KCl}$ and $\mathrm{LiCl}$ which is consistent with the FRET and FRET melting results.

Table 1. Kinetic and melting temperature $\left(T_{m}\right)$ parameters for hairpin to G-quadruplex conformational transition in the presence of indicated concentrations of TPE-MePy. ${ }^{\text {[a] }}$

\begin{tabular}{ccccc}
\hline $\begin{array}{c}\text { TPE-MePy } \\
\text { Conc. } \\
{\left[\times 10^{-6} \mathrm{M}\right]}\end{array}$ & $\begin{array}{c}k_{\mathrm{obs}} \\
{\left[\times 10^{-4} \mathrm{~s}^{-1}\right]}\end{array}$ & $\begin{array}{c}k_{\mathrm{obs} 2} \\
{\left[\times 10^{-4} \mathrm{~s}^{-1}\right]}\end{array}$ & $T_{m}\left({ }^{\circ} \mathrm{C}\right)$ & $\Delta T_{m}\left({ }^{\circ} \mathrm{C}\right)$ \\
\hline 0.00 & $1.33 \pm 0.12$ & $0.93 \pm 0.81$ & $92.22 \pm 1.38$ & \\
0.06 & $9.36 \pm 0.37$ & $0.79 \pm 0.68$ & $92.36 \pm 1.17$ & $0.14 \pm 2.46$ \\
0.12 & $12.4 \pm 1.48$ & $1.31 \pm 0.12$ & $92.14 \pm 1.72$ & $-0.08 \pm 0.51$ \\
0.25 & $8.52 \pm 7.61$ & $1.73 \pm 0.35$ & $93.49 \pm 0.35$ & $1.26 \pm 1.48$ \\
0.50 & $11.3 \pm 6.15$ & $1.69 \pm 0.43$ & $95.75 \pm 0.24$ & $3.52 \pm 1.53$ \\
1.00 & $19.4 \pm 0.10$ & $2.45 \pm 0.12$ & $96.59 \pm 1.01$ & $4.36 \pm 1.95$ \\
\hline
\end{tabular}

\footnotetext{
[a] $k_{o b s 1,} k_{o b s 2}$, and $\mathrm{T}_{\mathrm{m}}$ are averages from the triplicate experiments and the indicated errors are standard deviations.
} 

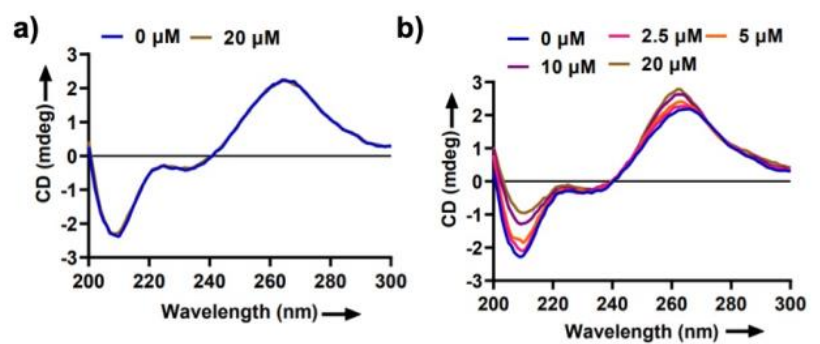

c)

\begin{tabular}{ccccc}
\hline $\begin{array}{c}\text { TPE-MePy Conc. } \\
{\left[\times 10^{-6} \mathrm{M}\right]}\end{array}$ & $\begin{array}{c}\mathrm{K}_{\mathrm{F}} \\
(265 \mathrm{~nm})\end{array}$ & $\mathrm{GQ}: \mathrm{Hp}$ & $\begin{array}{c}\mathrm{K}_{\mathrm{F}} \\
(210 \mathrm{~nm})\end{array}$ & $\mathrm{GQ}: \mathrm{Hp}$ \\
\hline 0.00 & 0.17 & $14.2: 85.8$ & 0.14 & $12.4: 87.6$ \\
2.50 & 0.49 & $33.0: 67.0$ & 0.27 & $21.4: 78.6$ \\
5.00 & 0.81 & $44.8: 55.2$ & 0.63 & $38.6: 61.4$ \\
10.00 & 6.61 & $86.8: 13.2$ & 3.61 & $78.3: 21.7$ \\
20.00 & - & $100: 0$ & - & $100: 0$ \\
\hline
\end{tabular}

Figure 3. Circular dichroism spectra of HpGQ-1 RNA in presence of indicated concentration of a) TPE-Py; b) TPE-MePy in $10 \mathrm{mM}$ lithium cacodylate buffer ( $\mathrm{pH}$ 7.2) containing $1 \mathrm{mM} \mathrm{MgCl}, 0.08 \%$ Tween-20 and $1 \mathrm{mM} \mathrm{KCl}$ at $25^{\circ} \mathrm{C}$. CD signals are average of three replicates. c) The equilibrium folding constant (KF) estimated at $265 \mathrm{~nm}$ and $210 \mathrm{~nm}$ wavelength at each TPE-MePy concentration.

Further, we have used changes in the CD ellipticity of a HpGQ-1 as a function of TPE-MePy concentration to determine the equilibrium folding constant (KF). CD has been used as a useful tool to follow the conformational transition in proteins as function of ligands to determine KF.[27] The KF of the hairpin and the GQ conformers at each TPE-MePy concentration was estimated using a reversible two-state equilibrium model. In two state equilibrium model we assumed that the intermediate triplex structure does not significantly affects the CD signal at equilibrium and that conversion of hairpin to GQ conformer is complete at $20 \square \mathrm{M}$ TPE-MePy (Supporting Information Equations 2,3) since we observed saturation in CD signals at this concentration. The KF values increased with increasing TPE-MePy concentration (Figure 3c) calculated for $210 \mathrm{~nm}$ and $265 \mathrm{~nm}$ wavelengths. The CD data suggested that in presence of 10 $\mu \mathrm{M}$ TPE-MePy, the GQ conformer predominated with KF values of 6.61 and of 3.61 at 265 $\mathrm{nm}$ and $210 \mathrm{~nm}$ respectively. The data suggests equilibrium shift towards GQ conformer by $72.6 \%$ (at $265 \mathrm{~nm}$ ) and $65.9 \%(210 \mathrm{~nm}$ ) relative to the absence of TPE-MePy respectively.

In human cells, the natural as well as synthetic RNA GQ structures located in the $5^{\prime}$-UTRs of mRNAs have been shown to suppress translation.[28] The small molecules that bind to GQ within 5'-UTR of eukaryotic mRNAs can interfere with the translation initiation, by blocking either the recruitment of ribosomes to mRNA or the ribosome scanning process.[28] Finally, 
we have performed reporter gene translation assays to evaluate the influence of TPE-MePy on the HPGQ mediated gene regulation. We have chosen two HpGQ sequences one is HpGQ-1 (Figure 1c) which was used in FRET assays and the second is HpGQ-2 derived from the 5' UTR of the humanTRF2 mRNA (Figure S9).[29] We also designed mutants i.e. HpGQ-1M and HpGQ-2M for HpGQ-1 and HpGQ-2 sequences respectively, in which we have disrupted the G-quadruplex structure but keeping the hairpin structure intact by replacing indicated guanine nucleotide with an adenosine (Figure S10, S11). The HpGQ sequences and its mutant were cloned into the 5' UTR of the Renilla luciferase reporter gene (Figure S12). The translation efficiency of these constructs in the presence of TPE derivatives were evaluated in a cell-free system consisting extracts from the rabbit reticulocyte lysate system.

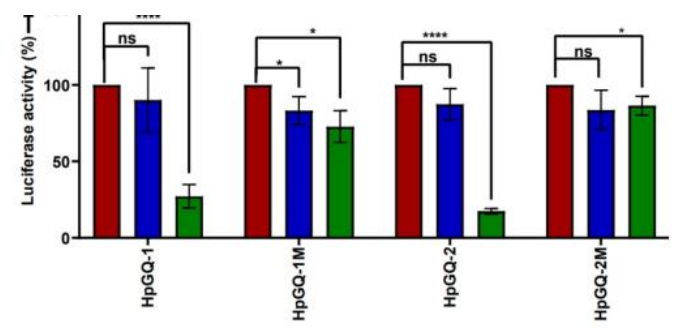

b)

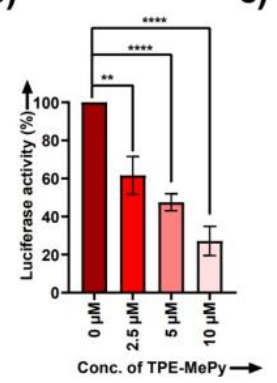

c)

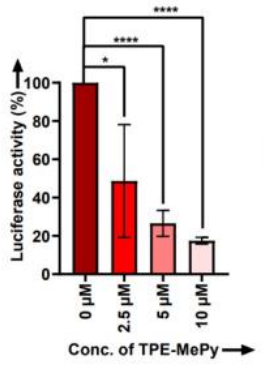

d)

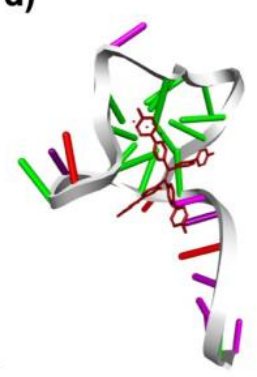

Figure 4. Effect of TPE derivatives on the in vitro translation efficiency measured by luciferase activity. a) Relative translation efficiencies of $\mathrm{HpGQ}$ and its mutants construct in $5^{\prime}$ UTR of mRNAs in the presence of indicated concentration of TPE derivatives. The luciferase activity for each HpGQ construct was normalized to the obtained luciferase activity in the absence of the TPE derivatives, which was set as $100 \%$. The experiments were performed in triplicates and the average values are shown with the standard errors. TPE-MePy concentration dependent relative translation efficiencies of b) HpGQ-1 and c) HpGQ-2 containing mRNAs respectively. Unpaired t-test was applied to obtain statistical $p$ values $c$ ) Docked structure of the TPE-MePy derivatives with HpGQ-1 RNA.

To perform luciferase assay, we have synthesized the reporter mRNAs, by in vitro transcription with T7 RNA polymerase. The reporter mRNAs were refolded with and without the $10 \square \mathrm{M}$ TPE derivatives and subsequently translated. The measured luciferase activity for each HpGQ construct was normalized to the luciferase activity obtained in the absence of the TPE derivatives (Figure 4). As a result, we found that in presence of $10 \square \mathrm{M}$ TPE-Py no significant differences in translation levels were observed for both the constructs (Figure 4a) which is 
consistent with our FRET and CD results. On the other hand, we have observed that TPEMePy derivatives significantly suppressed the translation efficiency upto $27.20 \%$ (3.7 fold) for HpGQ-1 and upto $17.46 \%$ (5.7 fold) for HpGQ-2 compared to the absence of ligand (Figure 4a). We did not observed any significant suppression in luciferase activity in mutant constructs suggesting the selective interaction of TPE-MePy with the GQ structures (Figure 4a). Upon gradually increasing the concentration of TPE-MePy from 0 to $10 \mu \mathrm{M}$ the translation efficiencies of both the constructs were suppressed in dose dependent manner (Figure 4b, 4c). These results indicate that the observed suppression in translation efficiency is due to the equilibrium shifts in favor of GQ conformer in the presence of TPE-MePy derivative.

In an effort to obtain further insights into the interaction between TPE derivatives and HpGQ structures at molecular level, we have performed molecular docking. Molecular docking has become an essential tool for screening of small molecule libraries against G-quadruplexes as well as to study structure-activity relationship for the selected small molecules toward the Gquadruplex binding.[30] Unfortunately, the structure is not solved for the hairpin-Gquadruplex forming RNA sequence. Thus we have chosen an NMR-refined structure of Monomeric Human CKIT-2 proto-oncogene promoter quadruplex (PDB ID: 1KF1) for the docking study. This parallel DNA GQ folded structure, which is believed to be similar to that of parallel RNA GQ was mutated using Discovery studio to generate RNA HpGQ structure for docking studies. The TPE derivatives were docked with the generated HpGQ-1 structure and the best binding configuration with the lowest energy was identified (Figures 4d, S13). The data suggests that both the TPE derivatives docks on the surface of the GQ structure mainly through $\pi-\pi$ stacking and van der waals interaction. Notably, the methyl group of TPE-MePy interacts with the 18th guanine residue of the HpGQ and Pyridine group of TPE-MePy interacts with the 13th guanine residues through carbon-hydrogen bond. However, TPE-Py does not show any carbon-hydrogen bond formation with HpGQ-1 (Figure S14). These observations suggest the role of methyl quaternary ammonium salt in binding and shifting of Hp-GQ equilibrium by stabilizing the GQ conformation.

In conclusion in this work, the two TPE derivatives TPE-Py and TPE-MePy were synthesized and their potential for shifting the Hp-GQ equilibrium in 5' UTR of proto-oncogene mRNAs was explored. The FRET and CD experiments suggested that the cationic TPE-MePy was able to shifts the Hp-GQ equilibrium towards the GQ conformer in concentration dependent 
manner. In presence of TPE-MePy the observed rate constant values for first and second folding step was increased up to 14.6 and 2.6-fold, respectively. The CD data also suggested that in presence of $10 \mu \mathrm{M}$ TPE-MePy the GQ conformer predominated with KF values of 6.61 at $265 \mathrm{~nm}$ and of 3.61 at $210 \mathrm{~nm}$. Notably, the unmethylated derivative TPE-Py doesn't show any influence on the Hp-GQ equilibrium. The FRET melting assay showed a strong stabilizing effect of TPE-MePy $\left(\Delta \mathrm{Tm}=4.36{ }^{\circ} \mathrm{C}\right)$. The molecular docking suggest that TPE-MePy molecule docks on the surface of the GQ structure mainly through $\pi-\pi$ stacking and van der waals interaction. The methyl group of TPE-MePy interacts with HpGQ through carbonhydrogen bond indicating the role of methyl quaternary ammonium salt in Hp-GQ transition. Finally, the in vitro luciferase assay demonstrated that the TPE-MePy derivatives supressed the translation upto $27.20 \%$ (3.7- fold) for HpGQ-1 and $17.46 \%$ (5.7-fold) for HpGQ-2 compared to the absence of ligand by shifting the hairpin-GQ equilibrium toward GQ conformers in synthetic constructs and in those containing 5' UTR of oncogenes. Taken together, our results indicate that Hp-GQ conformational equilibria should be considered as potential therapeutic targets for treating cancer. Furthermore, our results provide a strategy for the development of synthetic ligands to target the selective GQ structures based on their adjacent flanking sequences for therapeutic applications.

Keywords: Proto-oncogenes $\bullet$ Conformational equilibria $\bullet$ G-quadruplex $\bullet$ TPE

\section{References}

[1] J. L. Huppert, S. Balasubramanian, Nucleic Acids Res 2005, 33, 2908-2916.

[2] J. Moon, J. H. Han, D. Y. Kim, M. J. Jung, S. K. Kim, Biochem Biophys Rep 2015, 2, 29-35.

[3] aN. Kim, Curr Med Chem 2019, 26, 2898-2917; bM. M. Farhath, M. Thompson, S. Ray, A. Sewell, H. Balci, S. Basu, Biochemistry 2015, 54, 5533-5545.

[4] aA. Arora, M. Dutkiewicz, V. Scaria, M. Hariharan, S. Maiti, J. Kurreck, RNA 2008, 14, 1290-1296; bP. Agarwala, S. Pandey, K. Mapa, S. Maiti, Biochemistry 2013, 52, 15281538.

[5] aS. Artusi, M. Nadai, R. Perrone, M. A. Biasolo, G. Palu, L. Flamand, A. Calistri, S. N. Richter, Antiviral Res 2015, 118, 123-131; bU. Shankar, N. Jain, P. Majee, P. Kodgire, T. 
K. Sharma, A. Kumar, Front Genet 2020, 11, 935; cH. Han, L. H. Hurley, Trends Pharmacol Sci 2000, 21, 136-142.

[6] P. Agarwala, S. Pandey, S. Maiti, Org Biomol Chem 2015, 13, 5570-5585.

[7] S. Balaratnam, S. Basu, Biopolymers 2015, 103, 376-386.

[8] aS. Nakano, D. Miyoshi, N. Sugimoto, Chem Rev 2014, 114, 2733-2758; bT. Endoh, A. B. Rode, S. Takahashi, Y. Kataoka, M. Kuwahara, N. Sugimoto, Anal Chem 2016, 88, 1984-1989.

[9] A. B. Rode, T. Endoh, N. Sugimoto, Angew Chem Int Ed Engl 2016, 55, 14315-14319.

[10] aA. Bugaut, P. Murat, S. Balasubramanian, J Am Chem Soc 2012, 134, 19953-19956; bA. J. Zheng, A. Thermou, P. Guixens Gallardo, L. Malbert-Colas, C. Daskalogianni, N. Vaudiau, P. Brohagen, A. Granzhan, M. Blondel, M. P. Teulade-Fichou, R. P. Martins, R. Fahraeus, Life Sci Alliance 2022, 5.

[11] S. Pandey, P. Agarwala, S. Maiti, J Phys Chem B 2013, 117, 6896-6905.

[12] aC. K. Kwok, A. B. Sahakyan, S. Balasubramanian, Angew Chem Int Ed Engl 2016, 55, 8958-8961; bT. Endoh, N. Sugimoto, Molecules 2019, 24.

[13] aS. Pandey, P. Agarwala, G. G. Jayaraj, R. Gargallo, S. Maiti, Biochemistry 2015, 54, 7067-7078; bG. Mirihana Arachchilage, A. C. Dassanayake, S. Basu, Chem Biol 2015, 22, 262-272; cL. Pandolfini, I. Barbieri, A. J. Bannister, A. Hendrick, B. Andrews, N. Webster, P. Murat, P. Mach, R. Brandi, S. C. Robson, V. Migliori, A. Alendar, M. d'Onofrio, S. Balasubramanian, T. Kouzarides, Mol Cell 2019, 74, 1278-1290 e1279.

[14] A. Ghosh, M. K. Ekka, A. Tawani, A. Kumar, D. Chakraborty, S. Maiti, Biochemistry 2019, 58, 514-525.

[15] J. Figueiredo, T. Santos, A. Miranda, D. Alexandre, B. Teixeira, P. Simoes, J. LopesNunes, C. Cruz, Molecules 2021, 26.

[16] aD. D. La, S. V. Bhosale, L. A. Jones, S. V. Bhosale, ACS Appl Mater Interfaces 2018, 10, 12189-12216; bM. Salimimarand, D. D. La, M. A. Kobaisi, S. V. Bhosale, Sci Rep 2017, 7, 42898 .

[17] H. T. Feng, Y. X. Yuan, J. B. Xiong, Y. S. Zheng, B. Z. Tang, Chem Soc Rev 2018, $47,7452-7476$.

[18] L. Cheng, H. Zhang, Y. Dong, Y. Zhao, Y. Yu, L. Cao, Chem Commun (Camb) 2019, $55,2372-2375$.

[19] D. Wu, Z. Zhang, X. Yu, B. Bai, S. Qi, Front Chem 2021, 9, 817720.

[20] H. Tong, Y. Hong, Y. Dong, M. Haussler, J. W. Lam, Z. Li, Z. Guo, Z. Guo, B. Z. Tang, Chem Commun (Camb) 2006, 3705-3707. 
[21] aC. Kotras, M. Fossepre, M. Roger, V. Gervais, S. Richeter, P. Gerbier, S. Ulrich, M. Surin, S. Clement, Front Chem 2019, 7, 493; bY. Hong, M. Haussler, J. W. Lam, Z. Li, K. K. Sin, Y. Dong, H. Tong, J. Liu, A. Qin, R. Renneberg, B. Z. Tang, Chemistry 2008, 14, 64286437; cY. Hong, H. Xiong, J. W. Lam, M. Haussler, J. Liu, Y. Yu, Y. Zhong, H. H. Sung, I. D. Williams, K. S. Wong, B. Z. Tang, Chemistry 2010, 16, 1232-1245.

[22] aS. M. Flynn, S. T. George, L. White, W. Devonish, G. B. Takle, Biotechniques 1999, 26, 736-742, 744, 746; bJ. Rubio-Magnieto, F. Di Meo, M. Lo, C. Delcourt, S. Clement, P. Norman, S. Richeter, M. Linares, M. Surin, Org Biomol Chem 2015, 13, 2453-2463.

[23] aP. Alberti, J. L. Mergny, Proc Natl Acad Sci U S A 2003, 100, 1569-1573; bJ. L. Mergny, J. C. Maurizot, Chembiochem 2001, 2, 124-132.

[24] A. Y. Zhang, S. Balasubramanian, J Am Chem Soc 2012, 134, 19297-19308.

[25] A. De Rache, J. L. Mergny, Biochimie 2015, 115, 194-202.

[26] M. Vorlickova, I. Kejnovska, J. Sagi, D. Renciuk, K. Bednarova, J. Motlova, J. Kypr, Methods 2012, 57, 64-75.

[27] N. J. Greenfield, Nat Protoc 2006, 1, 2733-2741.

[28] A. Bugaut, S. Balasubramanian, Nucleic Acids Res 2012, 40, 4727-4741.

[29] D. Gomez, A. Guedin, J. L. Mergny, B. Salles, J. F. Riou, M. P. Teulade-Fichou, P. Calsou, Nucleic Acids Res 2010, 38, 7187-7198.

[30] aS. Roy, A. Ali, S. Bhattacharya, J Phys Chem B 2021, 125, 5489-5501; bJ. Dickerhoff, K. R. Warnecke, K. Wang, N. Deng, D. Yang, Int J Mol Sci 2021, 22. 